\title{
BIOINFORMATICS APPROACH ON ACTION AND MECHANISM OF BROMELAIN IN HEPATOCELLULAR CARCINOMA
}

\author{
SUSHMA S MURTHY ${ }^{1 *}$, BALA NARSAIAH T ${ }^{2}$ \\ ${ }^{1}$ Department of Biotechnology, JNTUA College of Engineering, Ananthapuram, Andhra Pradesh, India. ${ }^{2}$ Department of Chemical \\ Engineering, JNTUA College of Engineering, Ananthapuram, Andhra Pradesh, India. Email: sushmamicrobio@gmail.com
}

Received: 20 June 2021, Revised and Accepted: 03 August 2020

\section{ABSTRACT}

Objective: The objective of the study was to understand biomolecular interactions of Bromelain and its networking with p53 and $\beta$-catenin by a computational method of analysis in Hepatocellular carcinoma (HCC) condition.

Methodology: The protein interaction partners for p53 and $\beta$-catenin involved in the progression of HCC were collected from National Center for Biotechnology Information. We collected data points and standardized the data points for our data analysis from the public database. We used Cytoscape 3.8.2 version plug-in for constructing a Protein-Protein interaction network. We constructed a pathway network using Biorender.com.

Results: The protein interactions concerning p53 and $\beta$-catenin are identified and a network is constructed. A total of 18 and 34 nodes were identified which are involved in down-regulation and up-regulation of $\beta$-catenin and a total of 30 and 27 nodes for homosapiens are identified which are involved in the downregulation and upregulation of the p53 gene. We identified different pathways which trigger and impact the p53 and Wnt/ $\beta$ catenin signaling pathways as potential target sites for Bromelain to arrest the progression of cancer

Conclusion: In conclusion, our in silico studies anti-cancer activity of Bromelain in HCC relating its effect on apoptosis, cell differentiation, mesenchymal transition, p53 signaling, and Wnt/ $\beta$-catenin signaling pathways.

Keywords: Bromelain, Hepatocellular carcinoma, Protein-protein interaction-network, KEGG, p53, $\beta$-catenin.

(C) 2021 The Authors. Published by Innovare Academic Sciences Pvt Ltd. This is an open access article under the CC BY license (http://creativecommons.org/ licenses/by/4.0/) DOI: http://dx.doi.org/10.22159/ajpcr.2021v14i9.42661. Journal homepage: https://innovareacademics.in/journals/index.php/ajpcr

\section{INTRODUCTION}

Hepatocellular carcinoma (HCC) is one of the important types of cancer prevalent worldwide mainly in developing countries. HCC is the third most common cause of fatality in cancer-related diseases throughout the world. It is estimated that around 600,000 deaths per year resulted due to the effective invasive nature of the HCC cells which finally enter into the metastatic stage. The patients diagnosed as an advanced stage in HCC state have a low survival rate as there are no suitable curative therapies for such patients. Hence, there is an urgent need in addressing the effective treatment options to treat HCC patients. Further, evaluating the underlying molecular mechanisms in HCC provides alternate therapeutic strategies which help in achieving more favorable clinical outcomes with less toxicity and thereby reducing the overall morbidity rates [1].

Traditional medicine plays an important role in treating different types of cancer. The less toxic nature, safety, and efficacy of these phytocompounds makes them potent drugs for targeting cancer-related issues. Bromelain, a cysteine protease isolated from the stem of pineapple has a wide range of pharmacological significance [2]. In our previous studies, we report the anti-cancer property of bromelain in HepG2 cell lines using various in vitro models. We also report the drug impediment apoptosis by up-regulation of p53 and inhibition of invasive nature of cancer cells by down-regulation of a beta-catenin gene on treating with bromelain. Finally, we reported that bromelain is an effective phytocompound in controlling the progression of cancer cells in HCC conditions [2].

In the present study, an attempt is made in understanding the possible role of bromelain on molecular mechanisms and pathways underlying in HCC state. We used the protein-protein interaction (PPI) network approach, prediction of target sites of bromelain to understand its role as an anti-cancer agent. We also predicted the core modulatory network of bromelain targets p53 and beta-catenin which are significantly related to the process of apoptotic signaling pathway and regulation of cell death, proliferation, and survival. To our comprehension, this study is reported for the first time which deals with the prediction of the mechanism of Bromelain in HCC condition using the in-silicon approach. The study provides the promising implementation of bromelain as a novel drug in treating HCC.

\section{METHODOLOGY}

\section{Literature mining from public database}

The protein interaction partners for p53 and $\beta$-catenin involved in the progression of HCC were collected from [3] https://www.ncbi.nlm. nih.gov/pmc. The query gives for p53 was (p53 interaction HCC) and yielded 5474 hits and for $\beta$-catenin was ( $\beta$-catenin interaction HCC) and yielded 5458 hits. We limited our search for PMC articles and 10 years. To understand the role of Bromelain in other cancerous conditions, the query bromelain cancer was searched for PMC articles for 10 years.

\section{Data points collected}

We collected data points for our data analysis from the public database. The data points for network analysis are represented according to the sample format.

\section{Data standardization and enrichment}

We used public databases to standardization for our data points. National Center for Biotechnology Information (NCBI) gene database was used to get the complete functional analysis of a gene to elucidate its biological properties. The database was used to get information on gene symbols, ID, gene functioning like cellular and molecular process. Along with this Kyoto Encyclopedia of Genes and Genomes (KEGG) pathway was considered for functional analysis.

The data points are standardized as sample pattern as follows: 


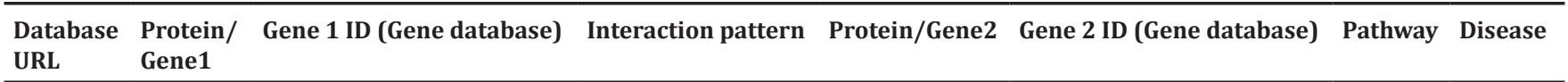

\section{PPI network visualization and data analysis}

We used Cytoscape 3.8.2 version plug-in for constructing the PPI network. The interactive networks for TP53, TP53-mut, $\beta$-catenin, $\beta$-catenin-mut, and bromelain were constructed based on the literature mining.

Construction of pathway for p53 and $\beta$-catenin

We constructed a pathway network using Biorender.com [4] (https:// biorender.com/) keeping the KEGG pathway as reference [5] (https:// www.genome.jp/kegg-bin/show_pathway?hsa05225) to understand the probable role of associated pathways in regulating the expression of p53 and $\beta$-catenin and indirectly affecting HCC diseased state.

\section{RESULTS}

\section{PPI network}

The Cytoscape plug-in version 3.8 .2 is used for performing PPI networking. The protein interactions with respect to p53 and $\beta$-catenin are identified and the network is constructed. A total of 18 and 34 nodes were identified which are involved in down-regulation and upregulation of $\beta$-catenin respectively. The 34 nodes identify are involved potentially in the progression of HCC (Figs. 1 and 2). Similarly, a total of 30 and 27 nodes for homosapiens are identified which is involved in the down-regulation and up-regulation of the p53 gene. On the other hand, 2 nodes and 1 node are identified for Mus musculus in downregulation and up-regulation of p53 (Figs. 3 and 4). The network analysis also includes the study of interaction patterns in PPI involved in the mutation of p53 and CTNNB1 protein and the networking is visualized by Cytoscape. The data points involved in targeting the mutant TP53 (TP53mut) and beta-catenin (CTNNB1mut) were collected. Potential 4 nodes and 3 nodes were identified in affecting mutation in $\beta$-catenin (Fig. 5) and p53 (Fig. 6). The data mining also reports the suppression of TP53mut by cinnamon oil and garlic oil. These identified genes act as potential nodes in regulating the disease progression in the HCC state.

Effect of Bromelain on various pathways affecting Wnt/ $\beta$-catenin signaling pathway and p53-dependent apoptotic pathway

The data points involved in the various pathways which affect Wnt/ $\beta$ catenin signaling pathway and p53-dependent apoptotic pathway are standardized. Keeping the KEGG pathway for HCC as reference (hsa05225), the Biorender tool was used to create different pathways. Wnt/ $\beta$-catenin signaling pathway is activated and inactivated form is shown in the figure (Figs. 7 and 8) and similarly, p53-dependent apoptotic pathway in is depicted in Figs. 9 and 10. The effect of Bromelain in these pathways which regulates p53 and $\beta$-catenin gene expressions is predicted in the HCC condition (Table 1).

\section{Effect of Bromelain on different types of cancer}

The data points for the anti-cancer activity of bromelain in different types of cancer are collected and standardized in MESH form. The MESH ID represents the following type of cancer on which bromelain has shown anti-cancer activity: D015179: Colon rectal cancer; C060192: Breast cancer; D010190: pancreatic cancer; D006258 HCC; C5876657: Gastric cancer; C587667 prostate cancer; D012878: Skin tumor; D001943 breast neoplasm; and C091991: human breast adenocarcinoma (Fig. 11).

\section{DISCUSSION}

Bromelain is an important dietary protein isolated from the stem and fruit of the pineapple. The studies have shown the significant effect of bromelain in treating allergy, inflammation, and cancer, as an immunomodulator which makes protein an important therapeutic

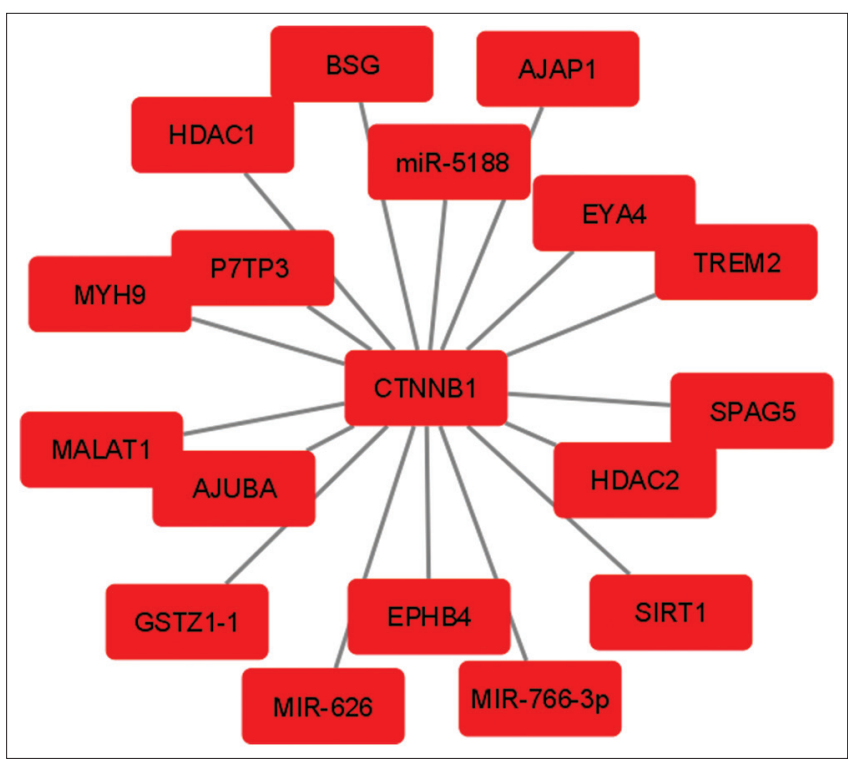

Fig. 1: Protein-protein interaction network involved in downregulation of CTNNB1 in hepatocellular carcinoma condition

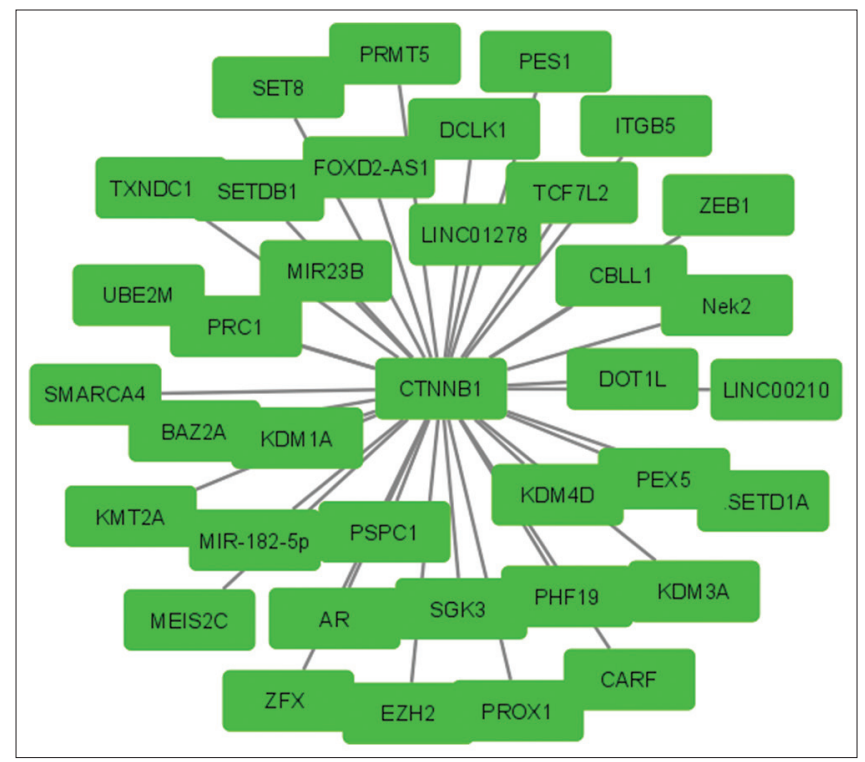

Fig. 2: Protein-protein interaction network involved in the upregulation of CTNNB1 in hepatocellular carcinoma condition

molecule. Previously our study reports the anti-cancer activity of bromelain in HepG2 cell lines and provides insight as an effective agent in treating HCC. We reported that in HCC, Bromelain exerts multiple effects in controlling the progression of cancer [6]. Our study reports that Bromelain is involved in inhibiting cell proliferation by arresting the cell cycle, induces cell death by triggering apoptotic pathway, reduces the invasiveness of cancer cells, and controls the progression of cancer to the metastatic stage in HepG2 cell lines. We report upregulation of p53 and down-regulation of $\beta$-catenin on treating with bromelain which indicates its role in modulating the associated molecules which are involved in their signaling pathways. 


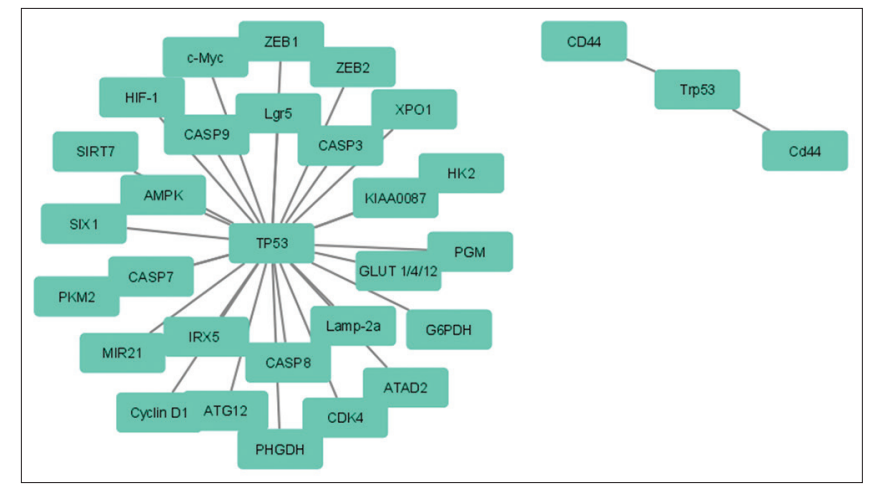

Fig. 3: Protein-protein interaction network involved in the downregulation ofTP53 in hepatocellular carcinoma condition

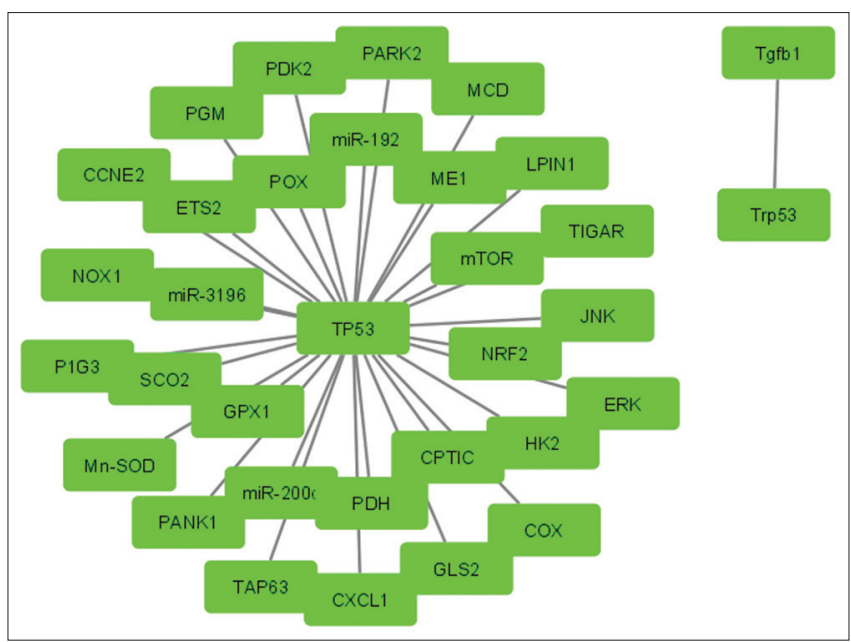

Fig. 4: Protein-protein interaction network involved in the upregulation ofTP53 in hepatocellular carcinoma condition

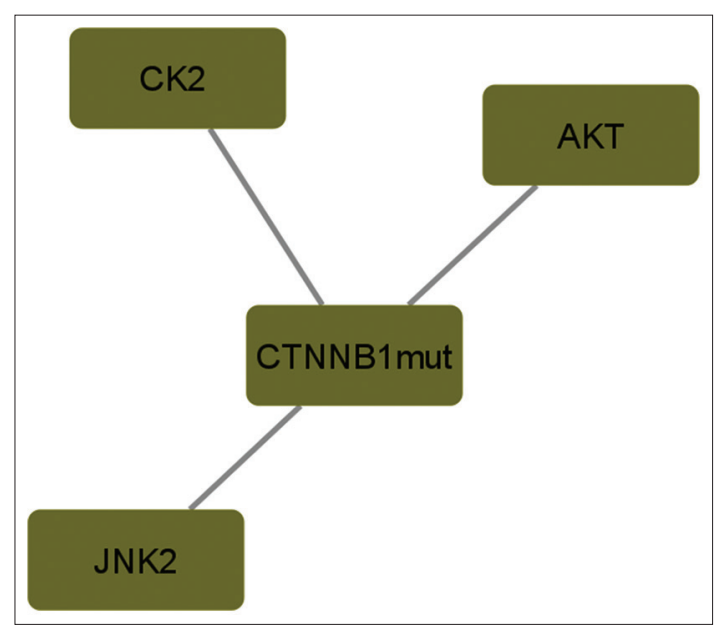

Fig. 5: Protein-protein interaction network involved in the upregulation of CTNNB1mut in hepatocellular carcinoma condition

The study reports IC10 $0.54 \mu \mathrm{M}$ and IC25 $0.72 \mu \mathrm{M}$ concentration of Bromelain can induce anti-cancer activity in HepG2 cell lines [6]. In the present study, we used the in silico approach to explore the molecular mechanism of bromelain in understanding its role in HCC from a systemic viewpoint.

Using the PPI-network approach we identified genes that play a key role in regulating signal transduction mechanism by p53. This

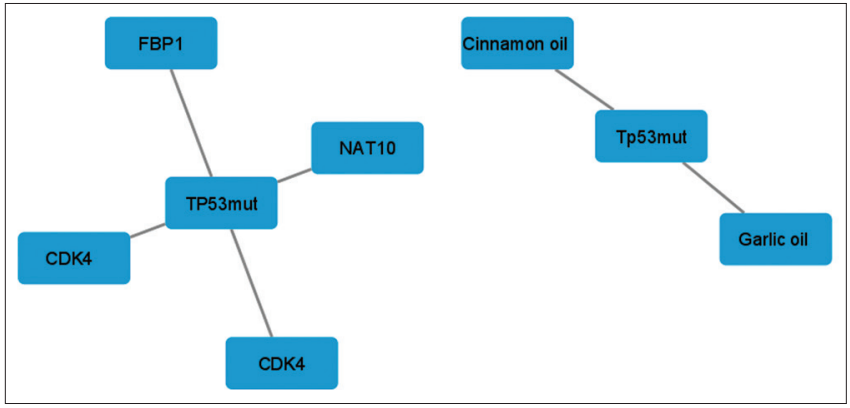

Fig. 6: Protein-protein interaction network involved in the upregulation ofTP53mut in hepatocellular carcinoma condition

Table 1: Predicted target sites of bromelain

\begin{tabular}{ll}
\hline Gene symbol & Protein name \\
\hline Hbp1 & $\begin{array}{l}\text { HMG-Box transcription factor 1 } \\
\text { Sterol regulatory element-binding transcription } \\
\text { factor } 1\end{array}$ \\
FOXP4 & Forkhead box protein P4 \\
GAS2 & Growth arrest-specific protein 2 \\
PARP & Poly (ADP-ribose) polymerase 1 \\
CASP3 & Caspase3 \\
CASP8 & Caspase8 \\
CASP9 & Caspase9 \\
Rab10 & Ras-related protein \\
ASPP2 & Apoptosis-stimulating of p53 protein 2 \\
AKT & Protein kinase B \\
TREM2 & Triggering receptor expressed on myeloid cells 2 \\
PI3K & Phosphatidylinositol 3-kinase \\
GST21 & Glutathione-S-Transferase \\
AJAP1 & Adherens Junction Associated protein 1 \\
ZEB1 & Zinc Finger E-box \\
GSK-3 $\beta$ & Glycogen synthase kinase3 \\
CKIa & Casein Kinase 1 alpha \\
BCL-2 & Apoptosis regulator BBcl2 \\
BAX & Bcl2-associated X protein \\
\hline
\end{tabular}

mechanism is involved in the regulation of cell death and brings about an intrinsic mode of the apoptotic signaling pathway. The PPI network also identified key genes which are involved in the regulation of $\beta$-catenin affecting the canonical pathway which brings regulation in the epithelial-mesenchymal transition (EMT), and affects the invasive property of the cancer cells. The expression of these genes is related to the overall progression of HCC affecting the disease-free survival rate. The study predicts that bromelain can act as a regulatory molecule where the study shows the cell cycle arrest and cell death leading to apoptotic pathway through TP53-dependent pathway in HepG2 cell lines [6].

The Wnt/ $\beta$-catenin pathway plays an important role in the progression of tumors at different stages in HCC conditions. The wnt $/ \beta$-catenin pathway also called a canonical pathway; activation and involvement form an important focus area for researchers in developing drug targeted therapies. Research shows that in most HCC patients there is a high expression of $\beta$-catenin which further enters into the canonical pathway. In normal conditions, the $\beta$-catenin level is highly regulated and is frequently degraded by the proteasomal machinery ensuring the inactivation or the off-state of the canonical pathway. In HCC condition in association with the other genes and pathways this mechanism is lost leading to the up-regulation and translocation of $\beta$-catenin from cytoplasm to nucleus wherein gets associated with the other factors and activates $\mathrm{Wnt} / \beta$-catenin signaling. On activation of this pathway, the downstream genes of the Wnt genes are expressed such as Cyclin D1, EMT, and c-Myc which are involved in the invasion, migration, and cell cycle activation process of cancer cells thereby enhancing 


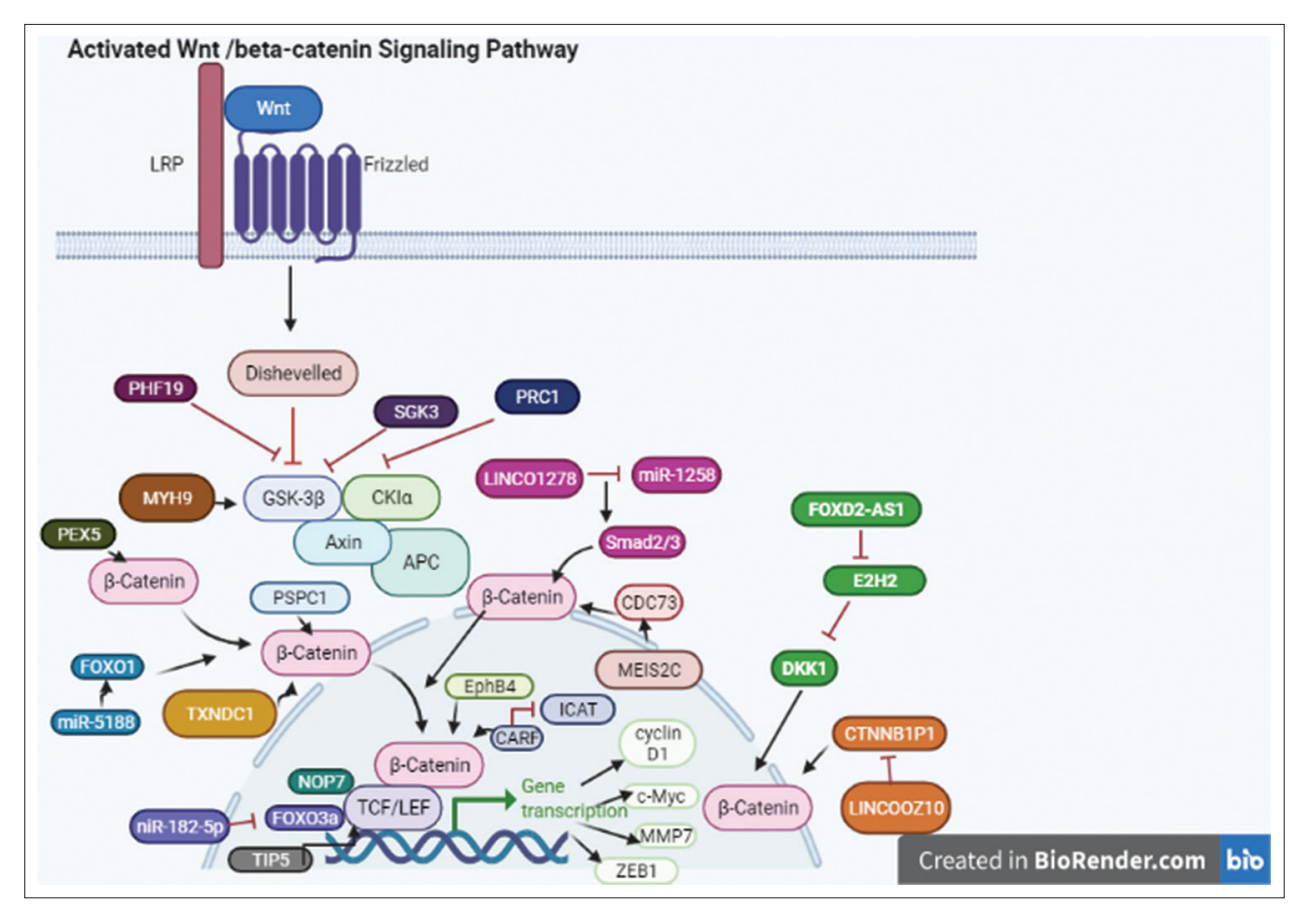

Fig. 7: Prediction of different types of pathways involved in activation of Wnt/ $\beta$-catenin pathway in hepatocellular carcinoma condition by in silico approach (The different pathways represented with different colors)

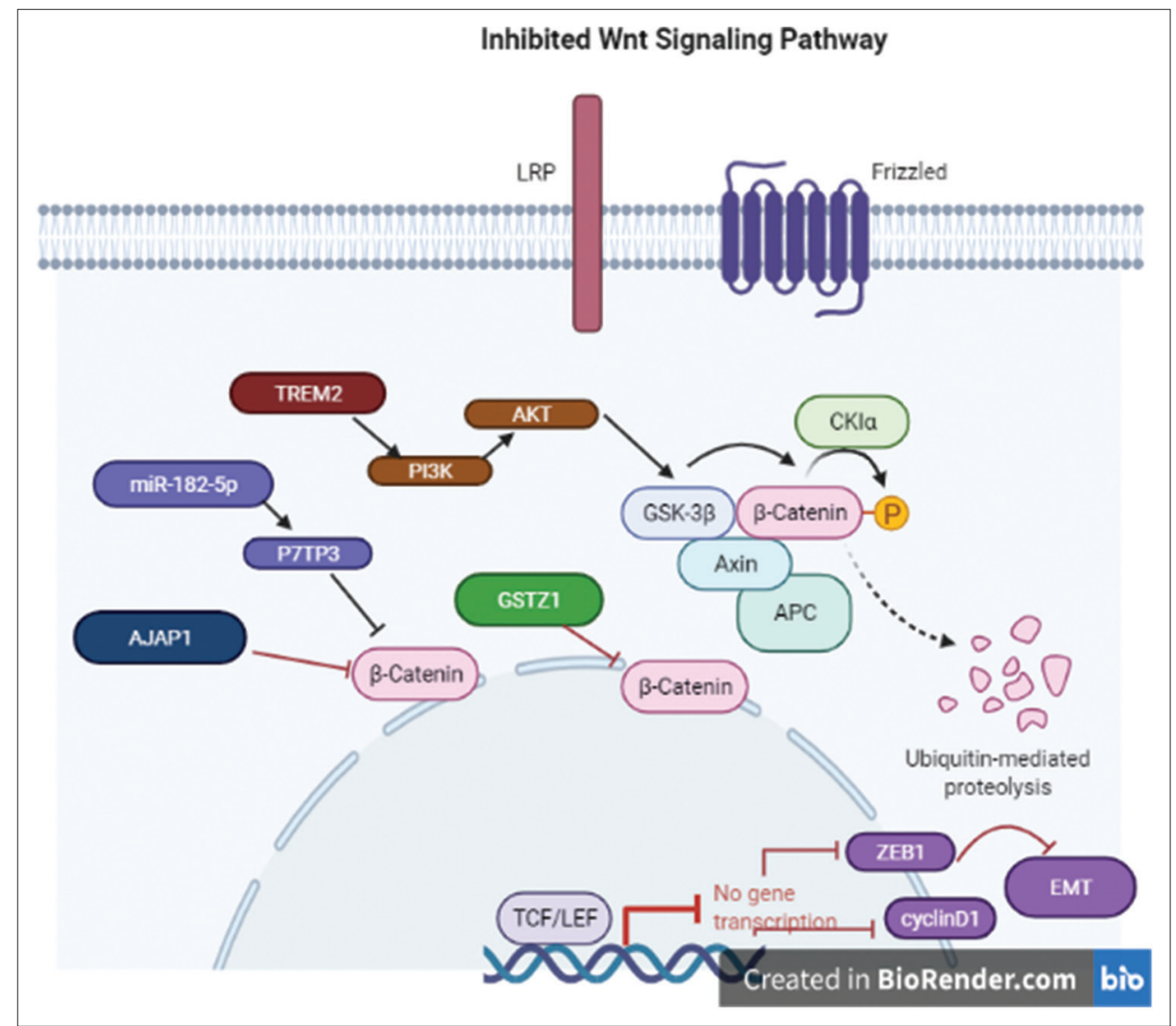

Fig. 8: Prediction of effect of bromelain on a different type of pathways involved in the inactivation of Wnt/ $\beta$-catenin pathway in hepatocellular carcinoma condition by in silico approach

the progression of HCC and leading to a metastatic state. With this background known $\beta$-catenin forms a key transcription factor to target and understand its involvement in the interaction with other molecules in upregulating the $\mathrm{Wnt} / \beta$-catenin pathway. 


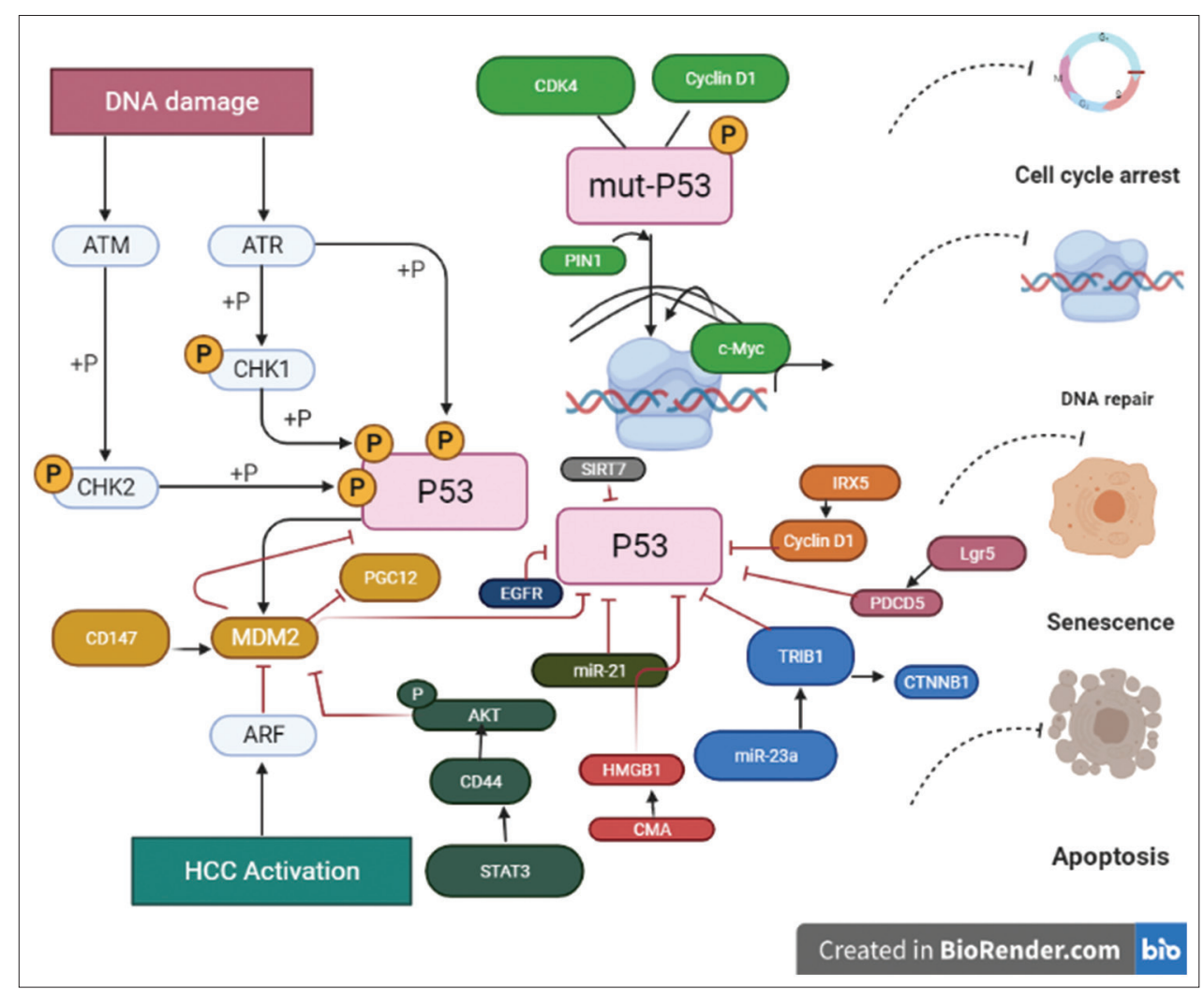

Fig. 9: Prediction of different types of pathways involved in the inactivation of p53-signaling pathway in hepatocellular carcinoma condition by in silico approach

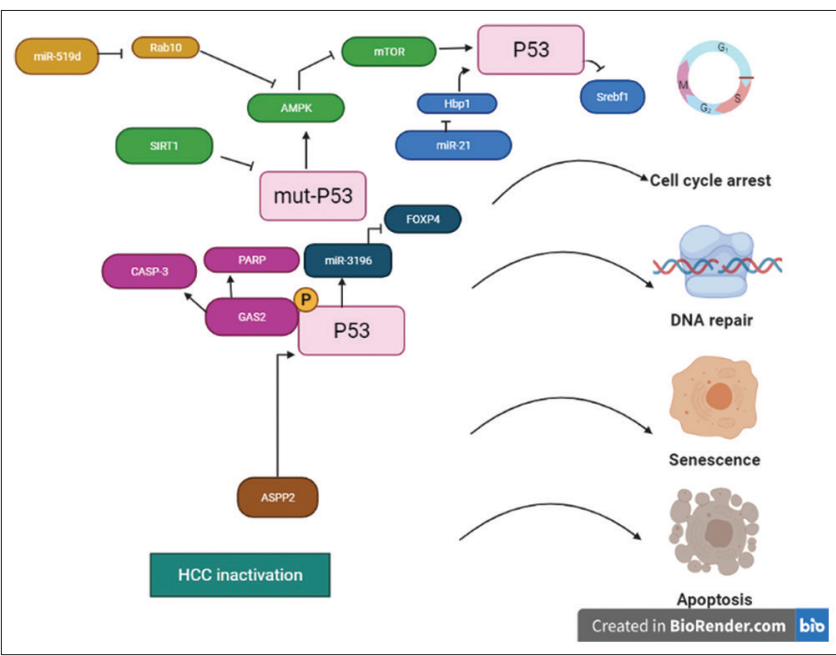

Fig. 10: Prediction of effect of Bromelain on a different type of pathways involved in the activation of $\mathrm{p} 53$ pathway in hepatocellular carcinoma condition by in silico approach

In our previous studies, an attempt has been made to study the effect of Bromelain, a phytocompound on the gene and protein expression of $\beta$-catenin and p53 in HepG2 cell lines using various in vitro tools. The study discussed and concludes that bromelain at its IC10 and IC25 concentration downregulates the gene expression of $\beta$-catenin and up-regulates p53 in HepG2 cell lines which further is correlated with the protein expression studies. In the present study using the in silico approach, an attempt is made to understand the interaction patterns of $\beta$-catenin and 553 with another protein that is involved in the apoptotic pathway mediated by p53 expression and Wnt/ $\beta$-catenin signaling pathway mediated by the expression of $\beta$-catenin. Finally, the probable role of Bromelain is predicted in p53 signal transduction and

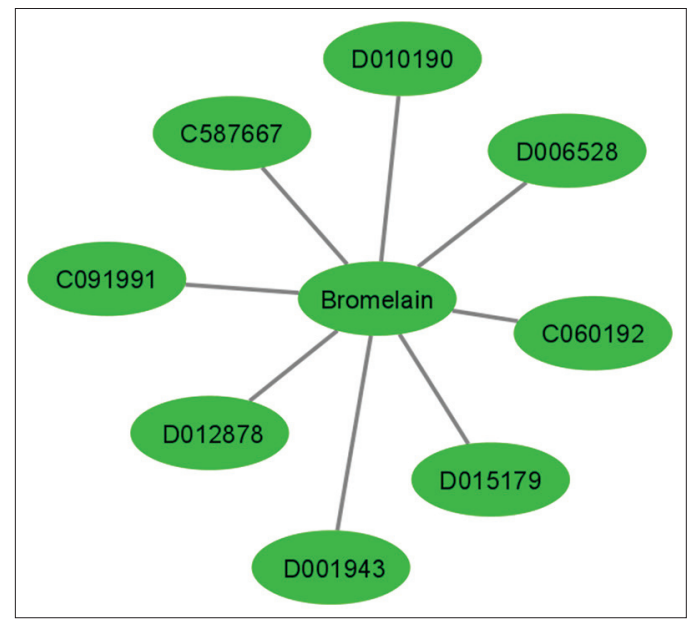

Fig. 11: Effect of bromelain on different forms of cancer which are represented in the MESH format

Wnt/ $\beta$-catenin signaling pathway in curtailing the spread of the disease through the bioinformatics approach.

The research has reported that some genes are involved in the downregulation of the $\beta$-catenin level, its activation, and interactions with the other molecules and blocks the Wnt $/ \beta$-catenin signaling pathway. This mechanism restricts the migration and invasive properties of cancer cells and inhibits the metastatic progression in HCC to a considerable extent. TREM2 or triggering receptor expresses on myeloid cell 2 activates PI3K which further induces phosphorylation of AKT. The active form of AKT triggers GSK-3 $\beta$ to activate $\beta$-catenin to undergo phosphorylation. The phosphorylated form of $\beta$-catenin is recognized by the proteasomal degradation system and degrades the $\beta$-catenin inhibiting pathway through the $\mathrm{PI} 3 \mathrm{~K} / \mathrm{Akt} / \beta$-catenin 
pathway [7]. The important mechanism for the activation of the canonical pathway is the translocation of $\beta$-catenin from the cytoplasm to the nucleus. This process is blocked by AJAP1 [8] and the effector response of this is downregulation of the ZEB1 gene which is involved in the EMT. P7TP3 down-regulates Wnt/ $\beta$-catenin signaling pathway and the gene are controlled by miR-182-5p [9]. The research predicted that DKK3 is the potential target for gene miR-626. The inactivation of DKK3 will reduce the Wnt/ $\beta$-catenin signaling and suppressed the spread of tumor in HCC conditions [10]. A similar effect is observed with the gene GSTZ1-1 [11].

The studies report different genes involved in different pathway mechanisms to activate $\beta$-catenin and regulate the $\mathrm{Wnt} / \beta$-catenin signaling pathway. The upstream factors affecting $\beta$-catenin can be effective receptors for targeting the drugs. A member of protein disulfide isomerase which is known as Thioredoxin domain-containing protein 12(TXNDC12) is involved in affecting the invasion and migration property of HCC cells in in vitro conditions. TXNDC12 up-regulates ZEB1, which further initiates EMT. ZEB1 is the major effector molecule of $\beta$-catenin. TXNDC12 binds with $\beta$-catenin and translocates it from the cytoplasm to the nucleus. In the nucleus, the $\beta$-catenin gets associated with the other genes and initiates the Wnt $/ \beta$-catenin signaling pathway in which one of the downstream genes is ZEB1. By ZEB1-mediated EMT, there is a rapid increase in the invasion and migratory rate of HCC cells [12]. The studies showed that activation of the Wnt/ $\beta$-catenin signaling pathway, interaction, and stabilization of $\beta$-catenin and TCF is important. PES1 gene is involved in this process through $\beta$-catenin/TCF signaling stabilizes the interaction of $\beta$-catenin and TCF [13]. The same type of study revealed that CARF is also involved in the stabilization process between $\beta$-catenin and TCF where CARF blocks ICAT from binding to $\beta$-catenin and helps $\beta$-catenin to interact with TCF [14]. A novel oncogene SGK3 up-regulates the progression of HCC by SGK3/ GSK-3 $\beta / \beta$-catenin pathway. Here, in the presence of PI3K inhibitor SGK3 phosphorylates GSK-3 $\beta$ on serine 9. This phosphorylated GSK$3 \beta$ inactivates the proteasomal degradation machinery by the level of $\beta$-catenin in the cytosol increases which under the influence of other genes translocates into the nucleus [15]. MiR-5188 interacts with FOXO1 which induces the translocation of $\beta$-catenin thereby activating Wnt genes EMT and c-Jun. c-Jun shows a positive feedback loop by involving in the expression of miR-5188 by the miR-5188-FOXO1/ $\beta$-catenin-cJun feedback loop pathway [16]. Studies have revealed that EphB4 is overexpressed in HCC tissues. EphB4 activates $\beta$-catenin to enter into a canonical pathway through EphB4/ $\beta$-catenin-dependent manner [17]. LINC01278 suppresses miR-1258 which results in the active expression of Smad2/3. Smad2/3 stabilizes $\beta$-catenin in the cytoplasm and helps in the process of translocation to the nucleus where $\beta$-catenin interacts with TCF and activates Wnt genes through $\beta$-catenin/TCF-4-LINC01278miR-1258-Smad2/3 feedback loop pathway [18]. MYH9 brings about ubiquitination and degradation of GSK-3 $\beta$ which in turn blocks the degradation of $\beta$-catenin. PHF19 is involved in the inactivation of the $\beta$-catenin degradation system, activates the $\beta$-catenin-TCF interaction, and facilitates the canonical pathway [19]. The gene PHF19 on activating Wnt/ $\beta$-catenin expresses an important inflammatory cytokine IL-6 through the $\beta$-catenin/IL6 signaling axis [20]. The mechanism of degradation of $\beta$-catenin proteasomal machinery and its stabilization is exhibited by ITGB5 through miR-185-ITGB5- $\beta$-catenin pathway [21], PSPC1 [22], PRC1 [23], and PEX5 [24] through Wnt/ $\beta$-catenin pathway activating Wnt gene thereby enhancing metastasis in HCC. FOXD2AS1 binds to EZH2 epigenetically and silences EZH2. The silencing of EZH2 further represses DKK1 and thereby the cells observe Wnt/ $\beta$ catenin signaling pathway [25]. MiR-182-5p inhibits the degradation system of $\beta$-catenin and negatively regulates FOXO3a activating Wnt/ $\beta$-catenin pathway [26]. MEIS2C potentially binds to CDC73 which is a dephosphorylated molecule that is involved in the activation of $\beta$-catenin [27]. LINC00210 binds to CTNNB1P1 which is an inhibitory molecule of $\beta$-catenin. The binding makes CTNNB1P1 lose its inhibitory property against $\beta$-catenin [28]. UBE2M is an oncogene involved in the translocation process of $\beta$-catenin from the cytoplasm to the nucleus where it activates the downstream expression of the gene cyclin D1 through $\beta$-catenin/cyclin D1 signaling [29]. TIP5 is the potential binding partner to TCFL2. The activated TCFL2 interacts and stabilizes with $\beta$-catenin. The research reports that in HCC patients there is overexpression of the miR-23b gene. The important direct target to this gene is ST7L. ST7L binds to the carboxyl-terminal region of AKT and inhibits the AKT/GSK3 $\beta / \beta$-catenin pathway [30]. All these mechanisms activate $\beta$-catenin to involve in Wnt/ $\beta$-catenin pathway and results in the progression of HCC. Therefore, the studies show that the activation of the Wnt/ $\beta$-catenin pathway is mainly by restricting the degradation of $\beta$-catenin by proteasomal degradation system, translocation of $\beta$-catenin accumulated in the cytoplasm to the nucleus, and enhancing the interaction of $\beta$-catenin with TCF. This mechanism forms the basis for the target to inhibit the activity of $\beta$-catenin.

The research reports that miR-21 blocks Hbp1 which further regulates p53 to block CCNB1, CCND1, Srebf1 [31], GAS2 triggers p53-GAS2Caspase cascade via p53 dependent apoptotic pathway [32], miR-3196 inhibits FOXP4 through p53-dependent, miR-3196-medicated-FOXP4 pathway [33] to induce cell death through p53 mediated apoptotic pathway in HCC condition.

Similarly, many genes are involved in the down-regulating p53 signal transduction process. CD 44 phosphorylates AKT which activates MDM2 termination of p53 through PI3k/AKT/MDM2 pathway. The studies reports, expression of miR-21 and CD147 degrades p53 [34]. Inhibition of p53 by EGFR by PI3K/AKT/ERT pathway [35], miR-23a activates TRIB1 which blocks p53 and activates CTNNB1 gene and regulates the expression of c-Myc, MMP7 and EMT [36], CMA down-regulates HMGB1 which in-turn down-regulates p53 [37], deacetylation of p53 by SIRT7 through SIRT7-p53 dependent pathway [38], Lgr5 target gene PDCD5 a programmed cell death protein which inhibits p53 via PDCD5/p53 signaling axis [39], IRX5 up-regulates cyclin D1 through p53 signaling pathway [40] inhibits the activity of p53. All the above-mentioned pathways inhibit the p53-mediated apoptosis in the HCC conditions.

In our in vitro studies, we observed that bromelain downregulated the expression of $\beta$-catenin and upregulation of p53 in HepG2 cell lines. The above-mentioned genes are predicted to be potential target sites for bromelain that might have inhibited the progression of HCC by affecting $\beta$-catenin in enhancing its degradation process or inhibiting the translocation process and thus downregulating Wnt $/ \beta$-catenin signaling pathway and up-regulating p53 mediated apoptotic pathway by activating the apoptotic proteins and the proteins involved in the cell cycle.

Bromelain is reported to up-regulate Casp-3, 8, and 9, PARP-1 in colon-rectal cancer through caspase-independent apoptotic pathway [41], downregulates GREM-1, IL-1 $\beta$, IL-4, NFkB1, PTGS2 in breast cancer [42], downregulates ACSL- 4 and upregulates ASCL4 in human colorectal cancer [43], Casp-3, Casp-9, Bax, and Trp53 is upregulated and Bcl-2, Ptgs2, Nfkb1, Nkbia, Mapk14, and Akt are down-regulated in skin tumor [44]. In the other study, Bromelain is reported to inhibit gastric carcinoma by cleaving TP53, upregulating CYCS, and downregulating BCL-2, AKT1, MUC1 through transcriptionindependent p53 apoptosis [45] which is a deviation from the present study. Overall the role of bromelain and its effect on the gene expression of other transcription factors are studied in various cancer conditions. This provides an insight into the significance of bromelain in apoptotic mediated cell death by interfering with the various types of apoptotic proteins in different cancerous conditions. Considering these facts we suggest that bromelain might have involved in exerting its effect on other genes apart from p53 and $\beta$-catenin in controlling the progression of HCC. In silico studies play a key role in predicting the suitable target site for the drug understudy in the cell [46].

\section{CONCLUSION}

Our in silico studies anti-cancer activity of bromelain in HCC relating its effect on apoptosis, cell differentiation, mesenchymal transition, p53 signaling, Wnt $/ \beta$-catenin signaling pathways. We here identify PPI 
network module which can be predicted as targets for bromelain which helps in the inhibition of progression of cancer to metastatic state in HCC condition. We also identified different pathways which trigger and impact the p53 and Wnt/ $\beta$-catenin signaling pathways as potential target sites for bromelain to arrest the progression of cancer. Further, biological experiments are needed to verify the present in silico study.

\section{ACKNOWLEDGMENT}

The authors are thankful to the management.

\section{AUTHOR CONTRIBUTION}

SSM studied the effect of bromelain on p53 and $\beta$-catenin protein expression and signaling pathway by in silico approach. Validation and writing were done by SSM and TBN.

\section{CONFLICT OF INTEREST}

The authors declare no conflict of interest.

\section{SOURCE OF FUNDING}

Self-funding.

\section{REFERENCES}

1. Li Z, Xu J, Cui H, Song J, Chen J, Wei J. Bioinformatics analysis of key biomarkers and potential molecular mechanisms in hepatocellular carcinoma induced by hepatitis B virus. Medicine 2020;99:e20302.

2. Sushma SM, Narsaiah B. Molecular insights into Bromelain application in industry and health care. Biosci Biotechnol Res Commun 2021;13:36-46.

3. Available from: https://www.ncbi.nlm.nih.gov/pmc/?term $=$ p53+and + beta+catenin+in+hcc. [Last accessed on 2021 Mar 03].

4. Available from: https://www.biorender.com. [Last accessed on 2021 Mar 07].

5. Available from: https:/www.genome.jp/kegg-bin/show pathway?hsa05225. [Last accessed on 2021 Mar 05].

6. Sushma SM, Narsaiah B. Cytotoxic effect of bromelain on $\mathrm{HepG}_{2}$ hepatocellular carcinoma cell line. Appl Biochem Biotechnol 2021;193:1873-97.

7. Tang W, Lv B, Yang B, Chen Y, Yuan F, Ma L, et al. TREM2 acts as a tumor suppressor in hepatocellular carcinoma by targeting the PI3K/ Akt/B-catenin pathway. Oncogenesis 2019;8:9.

8. Han J, Xie C, Pei T, Wang J, Lan Y, Huang K, et al. Deregulated AJAP1/ $\beta$-catenin/ZEB1 signaling promotes hepatocellular carcinoma carcinogenesis and metastasis. Cell Death Dis 2017;8:e2736.

9. Zhao J, Wang Y, Han M, Lu H, Chen X, Liu S, et al. P7TP3 inhibits tumor development, migration, invasion and adhesion of liver cancer through the Wnt//-catenin signaling pathway. Cancer Sci 2020;111:994-1007.

10. Zhu P, Liang H, Huang X, Zeng Q, Liu Y, Lv J, et al. Circular RNA Hsa circ 0004018 inhibits Wnt/ $\beta$-catenin signaling pathway by targeting microRNA-626/DKK3 in hepatocellular carcinoma. Onco Targets Ther 2020;13:9351-64.

11. Lei C, Wang Q, Tang N, Wang K. GSTZ1-1 downregulates Wnt/ $\beta$ catenin signalling in hepatocellular carcinoma cells. FEBS Open Bio 2020;10:6-17.

12. Yuan K, Xie K, Lan T, Xu L, Chen X, et al. TXNDC12 promotes EMT and metastasis of hepatocellular carcinoma cells via activation of $\beta$-catenin. Cell Death Differ 2020;27:1355-68.

13. Wu N, Zhao J, Yuan Y, Lu C, Zhu W, Jiang Q. NOP7 interacts with $\beta$-catenin and activates $\beta$-catenin/TCF signaling in hepatocellular carcinoma cells. Onco Targets Ther 2018;11:6369-76.

14. Fan X, Ma X, Cui L, Dang S, Qu J, Zhang J, et al. CARF activates beta-catenin/TCF signaling in the hepatocellular carcinoma. Oncotarget 2016;7:80404-14

15. Liu F, Wu X, Jiang X, Qian Y, Gao J. Prolonged inhibition of class I PI3K promotes liver cancer stem cell expansion by augmenting SGK3/ GSK-3 $\beta / \beta$-catenin signaling. J Exp Clin Cancer Res 2018;37:122.

16. Lin X, Zuo S, Luo R, Li Y, Yu G, Zou Y, et al. HBX-induced miR5188 impairs FOXO1 to stimulate $\beta$-catenin nuclear translocation and promotes tumor stemness in hepatocellular carcinoma. Theranostics 2019;9:7583-98.

17. Zhu M, Gong Z, Wu Q, Su Q, Yang T, Yu R, et al. Homoharringtonine suppresses tumor proliferation and migration by regulating EphB4mediated $\beta$-catenin loss in hepatocellular carcinoma. Cell Death Dis
2020;11:632

18. Huang WJ, Tian XP, Bi SX, Zhang SR, He TS, Song LY, et al. The $\beta$-catenin/TCF-4-LINC01278-miR-1258-Smad2/3 axis promotes hepatocellular carcinoma metastasis. Oncogene 2020;39:4538-50.

19. Lin X, Li AM, Li YH, Luo RC, Zou YJ, Liu YY, et al. Silencing MYH9 blocks HBx-induced GSK3 $\beta$ ubiquitination and degradation to inhibit tumor stemness in hepatocellular carcinoma. Signal Transduct Target Ther 2020;5:13.

20. Cai Z, Qian ZY, Jiang H, Ma N, Li Z, Liu LY, et al. hPCL3s promotes hepatocellular carcinoma metastasis by activating $\beta$-catenin signaling. Cancer Res 2018;78:2536-49.

21. Lin Z, He R, Luo H, Lu C, Ning Z, Wu Y, et al. Integrin- 35 , a miR185-targeted gene, promotes hepatocellular carcinoma tumorigenesis by regulating $\beta$-catenin stability. J Exp Clin Cancer Res 2018;37:17.

22. Lang YD, Chen HY, Ho CM, Shih JH, Hsu EC, Shen R, et al. PSPC1-interchanged interactions with PTK6 and $\beta$-catenin synergize oncogenic subcellular translocations and tumor progression. Nat Commun 2019; 10:5716.

23. Chen J, Rajasekaran M, Xia H, Zhang X, Kong SN, Sekar K, et al. The microtubule-associated protein PRC1 promotes early recurrence of hepatocellular carcinoma in association with the Wnt/ $\beta$-catenin signalling pathway. Gut 2016;65:1522-34.

24. Wen J, Xiong K, Aili A, Wang H, Zhu Y, Yu Z, et al. PEX5, a novel target of microRNA-31-5p, increases radioresistance in hepatocellular carcinoma by activating Wnt/ $\beta$-catenin signaling and homologous recombination. Theranostics 2020;10:5322-40.

25. Lei T, Zhu X, Zhu K, Jia F, Li S. EGR1-induced upregulation of lncRNA FOXD2-AS1 promotes the progression of hepatocellular carcinoma via epigenetically silencing DKK1 and activating Wnt/ $\beta$-catenin signaling pathway. Cancer Biol Ther 2019;20:1007-16.

26. Cao MQ, You AB, Zhu XD, Zhang W, Zhang YY, Zhang SZ, et al. miR$182-5 \mathrm{p}$ promotes hepatocellular carcinoma progression by repressing FOXO3a. J Hematol Oncol 2018;11:12

27. Guan L, Li T, Ai N, Wang W, He B, Bai Y, et al. MEIS2C and MEIS2D promote tumor progression via $\mathrm{Wnt} / \beta$-catenin and hippo/YAP signaling in hepatocellular carcinoma. J Exp Clin Cancer Res 2019;38:417.

28. Fu X, Zhu X, Qin F, Zhang Y, Lin J, Ding Y, et al. Linc00210 drives $\mathrm{Wnt} / \beta$-catenin signaling activation and liver tumor progression through CTNNBIP1-dependent manner. Mol Cancer 2018;17:73.

29. Zhang GC, Yu XN, Sun JL, Xiong J, Yang YJ, Jiang XM, et al. UBE2M promotes cell proliferation via the $\beta$-catenin/cyclin D1 signaling in hepatocellular carcinoma. Aging (Albany NY) 2020;12:2373-92.

30. Zhuang L, Wang X, Wang Z, Ma X, Han B, Zou H, et al. MicroRNA$23 \mathrm{~b}$ functions as an oncogene and activates $\mathrm{AKT} / \mathrm{GSK} 3 \beta / \beta$-catenin signaling by targeting ST7L in hepatocellular carcinoma. Cell Death Dis 2017;8:e2804.

31. Wu H, Ng R, Chen X, Steer CJ, Song G. MicroRNA-21 is a potential link between non-alcoholic fatty liver disease and hepatocellular carcinoma via modulation of the HBP1-p53-Srebp1c pathway. Gut 2016;65:1850-60.

32. Zhu RX, Cheng AS, Chan HL, Yang DY, Seto WK. Growth arrest-specific gene 2 suppresses hepatocarcinogenesis by intervention of cell cycle and p53-dependent apoptosis. World J Gastroenterol 2019;25:4715-26.

33. Qi W, Gao C, Zhang L, Gao Z, Sui J, Han C, et al. A p53-responsive microRNA, functions as a tumor suppressor in hepatocellular carcinoma by targeting FOXP4. Am J Cancer Res 2019;9:2665-78.

34. Cao H, Chen X, Wang Z, Wang L, Xia Q, Zhang W. The role of MDM2-p53 axis dysfunction in the hepatocellular carcinoma transformation. Cell Death Discov 2020;6:53.

35. Cioca A, Cimpean A, Ceausu R, Fit AM, Zaharie T, Al-Hajjar N, et al. Crosstalk between EGFR and p53 in hepatocellular carcinoma. Asian Pac J Cancer Prev 2014;15:8069-73.

36. Ye Y, Wang G, Wang G, Zhuang J, He S, Song Y, et al. The oncogenic role of tribbles 1 in hepatocellular carcinoma is mediated by a feedback loop involving microRNA-23a and p53. Front Physiol 2017;8:789.

37. Wu JH, Guo JP, Shi J, Wang H, Li LL, Guo B, et al. CMA downregulates p53 expression through degradation of HMGB1 protein to inhibit irradiation-triggered apoptosis in hepatocellular carcinoma. World J Gastroenterol 2017;23:2308-17.

38. Zhao J, Wozniak A, Adams A, Cox J, Vittal A, Voss J, et al. SIRT7 regulates hepatocellular carcinoma response to therapy by altering the p53-dependent cell death pathway. J Exp Clin Cancer Res 2019;38:252.

39. Ma Z, Guo D, Wang Q, Liu P, Xiao Y, Wu P, et al. Lgr5-mediated p53 repression through PDCD5 leads to doxorubicin resistance in hepatocellular carcinoma. Theranostics 2019;9:2967-83

40. Zhu L, Dai L, Yang N, Liu M, Ma S, Li C, et al. Transcription factorIRX5 promotes hepatocellular carcinoma proliferation and inhibits apoptosis by regulating the p53 signalling pathway. Cell 
Biochem Funct 2020;38:621-9.

41. Chang TC, Wei PL, Makondi PT, Chen WT, Huang CY, Chang YJ. Bromelain inhibits the ability of colorectal cancer cells to proliferate via activation of ROS production and autophagy. PLoS One 2019;14:e210274.

42. Mohamad NE, Abu N, Yeap SK, Alitheen NB. Bromelain enhances the anti-tumor effects of cisplatin on 4T1 breast tumor model in vivo. Integr Cancer Ther 2019;18:1534735419880258.

43. Park S, Oh J, Kim M, Jin EJ. Bromelain effectively suppresses Krasmutant colorectal cancer by stimulating ferroptosis. Anim Cells Syst (Seoul) 2018;22:334-40.
44. Kalra N, Bhui K, Roy P, Srivastava S, George J, Prasad S, et al. Regulation of $\mathrm{p} 53$, nuclear factor kappaB and cyclooxygenase-2 expression by bromelain through targeting mitogen-activated protein kinase pathway in mouse skin. Toxicol Appl Pharmacol 2008;226:30-7.

45. Amini A, Ehteda A, Moghaddam SM, Akhter J, Pillai K, Morris DL. Cytotoxic effects of bromelain in human gastrointestinal carcinoma cell lines (MKN45, KATO-III, HT29-5F12, and HT29-5M21). Onco Targets Ther 2013;6:403-9.

46. Kumar SS, Anjali T. In silico design and molecular docking studies of some 1, 2-benzisoxazole derivatives for their analgesic and antiinflammatory activity. Int J Curr Pharm Res 2017;9:133-6. 